\title{
Seismology of triple-mode classical Cepheids of the Large Magellanic Cloud
}

\author{
P. Moskalik ${ }^{1}$ and W. A. Dziembowski ${ }^{2,1}$ \\ ${ }^{1}$ Copernicus Astronomical Center, ul. Bartycka 18, 00-716 Warsaw, Poland \\ e-mail: pam@camk.edu.pl \\ 2 Warsaw University Observatory, Al. Ujazdowskie 4, 00-478 Warsaw, Poland
}

Received 5 October 2004/ Accepted 23 December 2004

\begin{abstract}
We interpret the three periods detected in OGLE LMC Cepheids SC3-360128 and SC5-338399 as corresponding to the first three overtones of radial pulsations. This interpretation imposes stringent constraints on parameters of the stars and on their evolutionary status, which could only be the first crossing of the instability strip. Evolutionary models reproducing measured periods exist only in a restricted range of metallicities $(Z=0.004-0.007)$. The models impose an upper limit to the extent of overshooting from the convective core. Absolute magnitude of each star is confined to a narrow interval, which allows us to derive a new estimate of the distance to the LMC. We obtain the value of $m-M$ ranging from 18.34 to 18 m.53 with a systematic difference between the two stars of about $0 \mathrm{~m} .13$. The rates of period change predicted by the models are formally in conflict with the derived observational limits, though the uncertainties of measured $\dot{P}$ may have been underestimated. If this discrepancy is confirmed, it would constitute a significant challenge to the stellar evolution theory.
\end{abstract}

Key words. stars: variables: Cepheids - stars: oscillations - stars: evolution - galaxies: Magellanic Clouds

\section{Introduction}

Triple-mode radial pulsators are extremely rare. For many years AC Andromedae, with the fundamental and the first two overtones simultaneously excited (Fitch \& Szeidl 1976), was the only known object of this type. Recently, two new triple-mode variables have been identified among short period Cepheids of the Large Magellanic Cloud (Moskalik et al. 2004; Moskalik \& Kołaczkowski 2005). Both stars, LMC SC3-360128 (hereafter Star 1) and LMC SC5-338399 (hereafter Star 2), have previously been known to pulsate in two radial modes, namely in the first and second overtones (Soszyński et al. 2000). The Moskalik \& Kołaczkowski (2005) analysis clearly shows presence of the third independent periodicity. The period ratio of $P_{3} / P_{2}=0.840$, the same in both Cepheids, points towards identification of the newly detected mode as a third radial overtone. The two stars are the first known pulsators with three consecutive radial overtones simultaneously excited.

Even if certain forms of pulsation are rare, it does not mean that they are unimportant. The best example is the role of double-mode Cepheid data in stimulating the revision of stellar opacities. Each measured period of an identified mode yields an accurate constraint on stellar parameters, and we have three such constraints for our objects. Another advantage is the membership of both Cepheids in the LMC. These stars have been and will be systematically monitored. Moreover, we have an estimate of their absolute luminosities. Unfortunately, this estimate is not precise, in particular because the distance to the LMC is still being debated. In fact, we will show that seismological analysis of the two triple-mode Cepheids yields an independent distance determination.

Our aim is to extract maximum information on the two stars from all available data. After summarizing observational information for them (Sect. 2), we focus on the inference from the values of measured periods, with an analysis in Sect. 3 similar to that of Kovács \& Buchler (1994) for AC And. We conclude, that both triple-mode Cepheids must be in the evolutionary phase of the Hertzsprung gap crossing and confront seismologically derived stellar parameters with predictions of the appropriate evolutionary models (Sect. 4). These models impose further constraints on the ranges of admissible stellar parameters. Section 5 is devoted to the LMC distance estimation implied by our models, while Sect. 6 compares theoretically predicted rates of period change with the limits inferred from observations. Finally, in Sect. 7 we summarize our conclusions and discuss future observations.

\section{Data}

Main observational properties of both triple-mode Cepheids are summarized in Table 1. Pulsation periods were determined from OGLE-II $I$-band photometry reduced with the DIA algorithm (Żebruń et al. 2001). All periods are accurate to better than $7 \times 10^{-6}$ day. Their errors are not quoted here as we 
Table 1. Triple-mode Cepheids in the LMC.

\begin{tabular}{|c|c|c|c|c|c|c|c|c|}
\hline OGLE No. & $\begin{array}{c}P_{1} \\
{[\text { day }]}\end{array}$ & $\begin{array}{c}P_{2} \\
{[\text { day }]}\end{array}$ & $\begin{array}{c}P_{3} \\
{[\text { day }]}\end{array}$ & $\begin{array}{c}V \\
{[\mathrm{mag}]}\end{array}$ & $\begin{array}{c}I \\
{[\mathrm{mag}]}\end{array}$ & $\begin{array}{c}E(B-V) \\
{[\mathrm{mag}]}\end{array}$ & $\begin{array}{c}(V-I)_{0} \\
{[\mathrm{mag}]}\end{array}$ & $\begin{array}{c}W_{I} \\
{[\mathrm{mag}]}\end{array}$ \\
\hline LMC SC3-360128 & 0.541279 & 0.436049 & 0.366301 & 17.53 & 16.98 & 0.134 & 0.38 & 16.13 \\
\hline LMC SC5-338399 & 0.579510 & 0.466624 & 0.392116 & 17.37 & 16.87 & 0.133 & 0.33 & 16.09 \\
\hline
\end{tabular}

believe they are smaller than uncertainties of the model periods and, therefore, inconsequential for our analysis. The mean $V$-band and $I$-band magnitudes were taken from Soszyński et al. (2000). The average $E(B-V)$ reddening for the field of each Cepheid (Udalski et al. 1999) was used to obtain dereddened $(V-I)_{0}$ colours. Standard extinction coefficients were adopted: $A_{V}=3.24 E(B-V), A_{I}=1.96 E(B-V)$. In the last column of the table we give Wessenheit index, $W_{I}=I-1.55 \times(V-I)$, which is an extinction insensitive brightness indicator (Madore \& Freedman 1991).

\section{Constraints on stellar parameters from pulsation periods}

The basic assumption behind our analysis was that the three periods detected in the triple-mode Cepheids correspond to the first three consecutive overtones of radial pulsations. The constraints on the stellar parmeters were derived by equating the three measured periods $P_{n, o}$ with periods calculated in plausible stellar models, $P_{n, c}$. For analysis in this section we used only envelope models, extending from the photosphere down to the temperature of $\log T=7.2$. This is the standard approach in studies of radial pulsations in giants. We checked that the calculated periods are sufficently insensitive to the location of the inner boundary of the envelope. The model periods depend on six parameters. These are: the mass $M$, luminosity $L$, effective temperature $T_{\text {eff }}$, the fractional abundances of hydrogen $X$, and of elements heavier than helium $Z$, and the mixing length parameter $\alpha_{\text {con }}$. For each star the pulsation data yield three equations of the form

$$
P_{n, c}\left(M, L, T_{\text {eff }}, X, Z, \alpha_{\text {con }}\right)=P_{n, o}
$$

but this is not enough to determine the values of all the unknown parameters. Theoretically, we could use mean magnitudes and colours of the stars, but these are uncertain. Therefore, we decided to find solutions for $M, L$ and $T_{\text {eff }}$ for selected $X, Z$, and $\alpha_{\text {con }}$ in admissible ranges, by relying only on the measured periods and requiring that all three modes are linearly unstable at specified parameters. Comparison with the observed mean colours and magnitudes was done a posteriori.

Our static stellar models, both envelope models used here, and the evolutionary models used in Sect. 4.2, were calculated with a modernized version of the Paczyński (1970) codes. The main improvements consisted in implementation of the OPAL equation-of-state and opacity data (Rogers et al. 1996; Iglesias \& Rogers 1996). Pulsation periods were calculated with a modified version of the Dziembowski (1977) code.

There are uncertainties in the calculated periods because we relied on the linear approximation. Unfortunately, we cannot estimate the nonlinear corrections to the periods. Comparison of periods derived from linear and from nonlinear codes has only been published for monoperiodic RR Lyr models (e.g. Kovács \& Buchler 1988) and long period Cepheid models (e.g. Moskalik \& Buchler 1991; Fokin 1994), so it is not clear how to scale these results to higher overtones in multiperiodic $\sim 3 M_{\odot}$ Cepheids.

Errors may also result from using the mixing length theory to calculate convective flux in our static models and from ignoring its Lagrangian perturbation in pulsating models. We believe that this is less essential and that our calculations made with $\alpha_{\text {con }}=0$ and $\alpha_{\text {con }}=1$ reflect the uncertainty well. The use of the Edddington approximation in the optically shallow layers is also an inadequacy but of secondary importance for the present application.

Simultaneous fit to three radial mode periods can be achieved for several different sets of parameters (e.g. AC And; Kovács \& Buchler 1994). In order to assure that we find all the solutions we applied the following procedure:

1. First, we selected chemical composition $(X, Z)$ and mixing length $\alpha_{\text {con }}$. Keeping these three parameters fixed, we calculated a grid of stellar models covering mass range of $2<$ $M / M_{\odot}<5$ and luminosity range of $2<\log \left(L / L_{\odot}\right)<3$.

2. At every point of the $(M, L)$ grid we found the model fitting the observed period of the first overtone, $P_{1, o}$. This can be easily achieved by adjusting the last remaining parameter of the model, its effective temperature. The solution for $T_{\text {eff }}$ is unique. The model fitting $P_{1}$ does not necessarily reproduce the other two periods. We calculated period mismatches $\Delta P_{2}=P_{2, c}-P_{2, o}$ and $\Delta P_{3}=P_{3, c}-P_{3, o}$.

3. We found the lines of $\Delta P_{2}=0$ on the $(M, L)$ plane. Models located on these lines reproduce both $P_{1}$ and $P_{2}$. Similarly, we found the lines of $\Delta P_{3}=0$. The values of $(M, L)$ at which the two types of lines cross each other define the models which simultaneously reproduce all three periods.

In Fig. 1 we display the results of the procedure as applied to Star 2, for $\alpha_{\text {con }}=0, X=0.70$, and three different metallicity values $(Z=0.009,0.007,0.005)$. The filled and open circles define the lines of $\Delta P_{2}=0$ and $\Delta P_{3}=0$, respectively. The dotted area in each plot corresponds to models with all three modes linearly unstable. We recall here that in the absence of resonances (as in our case), the linear excitation of all modes is the necessary condition for triple-mode pulsations to exist. Among all models matching the observed periods, only those located in the dotted area correspond to acceptable solutions.

As we see from Fig. 1, the problem of matching periods of a triple-mode Cepheid can have up to three different solutions, called $A, B$, and $C$. Solution $A$ exists for all choices of $X, Z$, 

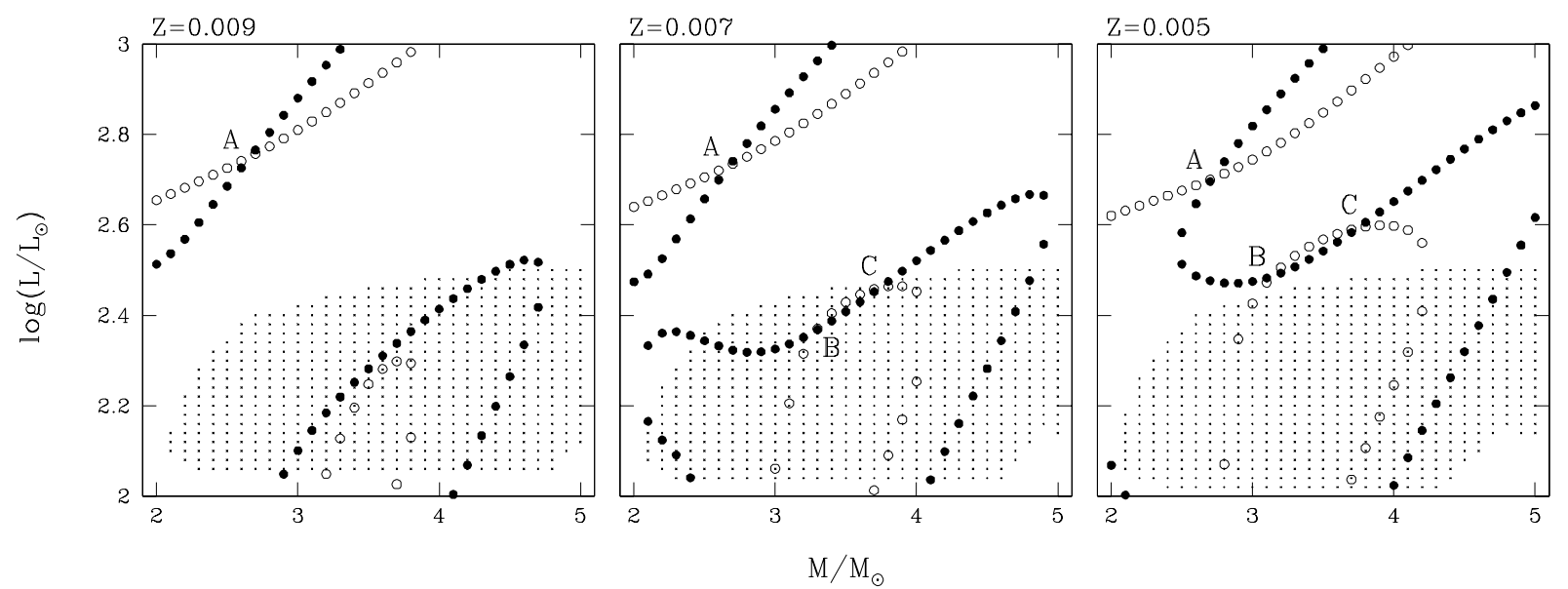

Fig. 1. Simultaneous fit to three pulsation periods observed in Star 2. Three panels display models calculated for $\alpha_{\text {con }}=0 ; X=0.70$; and metallicities of $Z=0.009, Z=0.007$ and $Z=0.005$. Filled and open circles denote lines of $\Delta P_{2}=0$ and $\Delta P_{3}=0$, respectively. Different triple-mode solutions are marked $A, B$ and $C$. Dotted area is the domain where all three modes are linearly unstable.

Table 2. Seismic envelope models for Star 1.

\begin{tabular}{|c|c|c|c|c|c|c|c|}
\hline$\alpha_{\mathrm{con}}$ & $X$ & $Z$ & $M / M_{\odot}$ & $\log \left(L / L_{\odot}\right)$ & $\begin{array}{c}\log T_{\text {eff }} \\
{[\mathrm{K}]}\end{array}$ & $\begin{array}{c}(V-I) \\
{[\mathrm{mag}]}\end{array}$ & $\begin{array}{c}m-M \\
{[\mathrm{mag}]}\end{array}$ \\
\hline \multirow[t]{2}{*}{0} & 0.74 & 0.006 & 3.29 & 2.311 & 3.814 & 0.50 & 18.43 \\
\hline & & & 3.63 & 2.403 & 3.829 & 0.43 & 18.52 \\
\hline \multirow[t]{2}{*}{0} & 0.74 & 0.005 & 3.15 & 2.358 & 3.828 & 0.44 & 18.42 \\
\hline & & & (3.67 & 2.487 & 3.849) & & \\
\hline \multirow[t]{2}{*}{1} & 0.74 & 0.007 & 3.48 & 2.299 & 3.807 & 0.52 & 18.47 \\
\hline & & & 3.67 & 2.359 & 3.818 & 0.48 & 18.52 \\
\hline \multirow[t]{2}{*}{1} & 0.74 & 0.006 & 3.21 & 2.316 & 3.817 & 0.48 & 18.42 \\
\hline & & & 3.68 & 2.423 & 3.834 & 0.41 & 18.53 \\
\hline \multirow[t]{2}{*}{1} & 0.74 & 0.005 & 3.14 & 2.376 & 3.833 & 0.42 & 18.41 \\
\hline & & & 3.59 & 2.461 & 3.845 & 0.37 & 18.51 \\
\hline \multirow[t]{2}{*}{1} & 0.74 & 0.004 & 3.14 & 2.433 & 3.847 & 0.36 & 18.42 \\
\hline & & & (3.60 & 2.547 & $3.865)$ & & \\
\hline \multirow[t]{2}{*}{0} & 0.70 & 0.008 & 3.28 & 2.195 & 3.787 & 0.60 & 18.39 \\
\hline & & & 3.48 & 2.263 & 3.800 & 0.55 & 18.45 \\
\hline \multirow[t]{2}{*}{0} & 0.70 & 0.007 & 3.10 & 2.215 & 3.795 & 0.57 & 18.37 \\
\hline & & & 3.63 & 2.366 & 3.822 & 0.46 & 18.50 \\
\hline \multirow[t]{2}{*}{0} & 0.70 & 0.006 & 2.98 & 2.279 & 3.813 & 0.50 & 18.36 \\
\hline & & & 3.66 & 2.437 & 3.839 & 0.39 & 18.52 \\
\hline \multirow[t]{2}{*}{0} & 0.70 & 0.005 & 2.88 & 2.346 & 3.832 & 0.42 & 18.35 \\
\hline & & & (3.66 & 2.506 & $3.856)$ & & \\
\hline \multirow[t]{2}{*}{1} & 0.70 & 0.007 & 3.21 & 2.240 & 3.799 & 0.56 & 18.40 \\
\hline & & & 3.67 & 2.388 & 3.827 & 0.44 & 18.51 \\
\hline \multirow[t]{2}{*}{1} & 0.70 & 0.006 & 2.96 & 2.300 & 3.819 & 0.47 & 18.36 \\
\hline & & & 3.65 & 2.440 & 3.840 & 0.39 & 18.51 \\
\hline \multirow[t]{2}{*}{1} & 0.70 & 0.005 & 2.90 & 2.362 & 3.836 & 0.40 & 18.35 \\
\hline & & & (3.61 & 2.487 & 3.852) & & \\
\hline \multirow[t]{2}{*}{1} & 0.70 & 0.004 & 2.86 & 2.410 & 3.848 & 0.35 & 18.35 \\
\hline & & & (3.63 & 2.575 & 3.873) & & \\
\hline
\end{tabular}

and $\alpha_{\text {con }}$, but all three modes are always linearly stable, so this solution will not be discussed any further. Solutions $B$ and $C$ can exist only for metallicities below a certain critical value. We may see in Fig. 1 that for $Z=0.009$, the lines $\Delta P_{2}=0$ and $\Delta P_{3}=0$ are close to each other but do not cross. As the value of $Z$ is lowered, the two lines come closer and finally cross each other, generating solutions $B$ and $C$. For the case displayed in Fig. 1, this happens for $Z \sim 0.0082$. When $Z$ is lowered further, first solution $C$ and then solution $B$ migrate out of the dotted area. This sets a lower limit for the metallicity. Below this limit 
Table 3. Seismic envelope models for Star 2.

\begin{tabular}{|c|c|c|c|c|c|c|c|}
\hline$\alpha_{\mathrm{con}}$ & $X$ & $Z$ & $M / M_{\odot}$ & $\log \left(L / L_{\odot}\right)$ & $\begin{array}{c}\log T_{\text {eff }} \\
{[\mathrm{K}]}\end{array}$ & $\begin{array}{c}(V-I) \\
{[\mathrm{mag}]}\end{array}$ & $\begin{array}{c}m-M \\
{[\mathrm{mag}]}\end{array}$ \\
\hline 0 & 0.70 & 0.008 & $\begin{array}{l}3.45 \\
3.65\end{array}$ & $\begin{array}{l}2.333 \\
2.382\end{array}$ & $\begin{array}{l}3.807 \\
3.815\end{array}$ & $\begin{array}{l}0.52 \\
0.49\end{array}$ & $\begin{array}{l}18.52 \\
18.57\end{array}$ \\
\hline 0 & 0.70 & 0.007 & $\begin{array}{l}3.29 \\
3.74\end{array}$ & $\begin{array}{l}2.367 \\
2.461\end{array}$ & $\begin{array}{l}3.818 \\
3.833\end{array}$ & $\begin{array}{l}0.48 \\
0.42\end{array}$ & $\begin{array}{l}18.50 \\
18.60\end{array}$ \\
\hline 0 & 0.70 & 0.006 & $\begin{array}{r}3.21 \\
(3.74\end{array}$ & $\begin{array}{l}2.424 \\
2.525\end{array}$ & $\begin{array}{l}3.834 \\
3.848)\end{array}$ & 0.41 & 18.49 \\
\hline 1 & 0.70 & 0.008 & $\begin{array}{l}3.41 \\
3.74\end{array}$ & $\begin{array}{l}2.337 \\
2.416\end{array}$ & $\begin{array}{l}3.809 \\
3.823\end{array}$ & $\begin{array}{l}0.51 \\
0.46\end{array}$ & $\begin{array}{l}18.51 \\
18.58\end{array}$ \\
\hline 1 & 0.70 & 0.007 & $\begin{array}{l}3.27 \\
3.75\end{array}$ & $\begin{array}{l}2.385 \\
2.469\end{array}$ & $\begin{array}{l}3.824 \\
3.836\end{array}$ & $\begin{array}{l}0.45 \\
0.40\end{array}$ & $\begin{array}{l}18.49 \\
18.59\end{array}$ \\
\hline 1 & 0.70 & 0.006 & $\begin{array}{l}3.25 \\
3.67\end{array}$ & $\begin{array}{l}2.438 \\
2.504\end{array}$ & $\begin{array}{l}3.837 \\
3.845\end{array}$ & $\begin{array}{l}0.40 \\
0.36\end{array}$ & $\begin{array}{l}18.50 \\
18.59\end{array}$ \\
\hline 1 & 0.70 & 0.005 & $\begin{array}{r}3.26 \\
(3.64\end{array}$ & $\begin{array}{l}2.489 \\
2.563\end{array}$ & $\begin{array}{l}3.849 \\
3.860)\end{array}$ & 0.35 & 18.51 \\
\hline 0 & 0.72 & 0.007 & $\begin{array}{l}3.52 \\
3.66\end{array}$ & $\begin{array}{l}2.397 \\
2.430\end{array}$ & $\begin{array}{l}3.822 \\
3.827\end{array}$ & $\begin{array}{l}0.46 \\
0.44\end{array}$ & $\begin{array}{l}18.54 \\
18.58\end{array}$ \\
\hline 0 & 0.72 & 0.006 & $\begin{array}{r}3.38 \\
(3.72\end{array}$ & $\begin{array}{l}2.438 \\
2.508\end{array}$ & $\begin{array}{l}3.834 \\
3.845)\end{array}$ & 0.41 & 18.53 \\
\hline 1 & 0.72 & 0.007 & $\begin{array}{l}3.42 \\
3.73\end{array}$ & $\begin{array}{l}2.399 \\
2.456\end{array}$ & $\begin{array}{l}3.824 \\
3.832\end{array}$ & $\begin{array}{l}0.45 \\
0.42\end{array}$ & $\begin{array}{l}18.53 \\
18.60\end{array}$ \\
\hline 1 & 0.72 & 0.006 & $\begin{array}{l}3.45 \\
3.59\end{array}$ & $\begin{array}{l}2.458 \\
2.482\end{array}$ & $\begin{array}{l}3.838 \\
3.841\end{array}$ & $\begin{array}{l}0.39 \\
0.38\end{array}$ & $\begin{array}{l}18.54 \\
18.57\end{array}$ \\
\hline
\end{tabular}

solutions $B$ and $C$ exist but do not correspond to triple-mode pulsations, because at least one of the modes is linearly stable. For the case of Fig. 1 this lower limit is $Z \sim 0.0056$.

We searched for solutions for both triple-mode Cepheids, in the range of metallicities from $Z=0.004$ to 0.010 (in some cases to 0.020 ) and of hydrogen abundances from $X=0.70$ to 0.74 . For each chemical composition we calculated models with $\alpha_{\text {con }}=0$ and with $\alpha_{\text {con }}=1$. The results of this survey are summarized in Table 2 for Star 1 and in Table 3 for Star 2. We listed only those combinations of $X, Z$, and $\alpha_{\text {con }}$ for which at least one acceptable solution exists. For low metallicities, solution $C$ may already fall outside the "all-modes-unstable" domain, in which case it was given in parenthesis. For all acceptable solutions we provided the values of $(V-I)$ colour and the distance modulus to the star, $m-M$. The latter was obtained by comparing Wessenheit index, $W_{I}$, derived from models with the mean observed values, as given in Table 1 . The conversion from model parameters to $V$-band and $I$-band magnitudes was done with tabular data based on Kurucz's (1998) stellar atmosphere models.

The triple-mode pulsations strongly constrain parameters of the studied Cepheids. We find that metallicities of both stars have to be in the range of $Z=0.0035-0.008$, with limiting values somewhat dependent on $X$ and assumed $\alpha_{\text {con }}$. For Star 1 , fitting the observed periods is possible for all values of $X$, but for Star 2 hydrogen abundance of $X \leq 0.72$ is required. The inferred stellar masses are limited to ranges of $2.8<M / M_{\odot}<3.7$ for Star 1 and $3.2<M / M_{\odot}<3.8$ for Star 2 .
The respective distance moduli are in the ranges of 18. $35-18.53$ and $18.49-18.60$.

In the next section we will show that the constraints imposed by triple-mode pulsations can be considerably tightened by combining the seismic envelope models with the appropriate evolutionary tracks.

\section{Evolutionary models}

\subsection{Evolutionary status of the stars}

The very short periods and low luminosities of the triple-mode Cepheids cannot be explained with current models of stars undergoing core helium burning. Whether or not a star enters the instability strip during this evolutionary phase depends on the extent of the blue loop, determined mainly by the mass and the metallicity of the star. Alibert et al. (1999) found that at $Z=0.004$ their models with masses of 3.0-3.5 $M_{\odot}$ do enter the instability strip; however, the minimum value of $P_{1}$, which occurs for $M=3 M_{\odot}$, is about 0.9 . At $Z=0.01$ they found models with blue loop extending to the instability strip only at $M \geq 4 M_{\odot}$, which naturally have much higher luminosities and longer pulsation periods than our stars. Alibert et al. (1999) assumed negligible overshooting in their calculations.

The effect of finite overshooting was taken into account in an extensive set of models by Girardi et al. (2000). In this case, the parameters we derived for the triple-mode Cepheids are even more difficult to reconcile with models in the helium burning phase. At $Z=0.004$ and $M=3 M_{\odot}$ the loop does not 

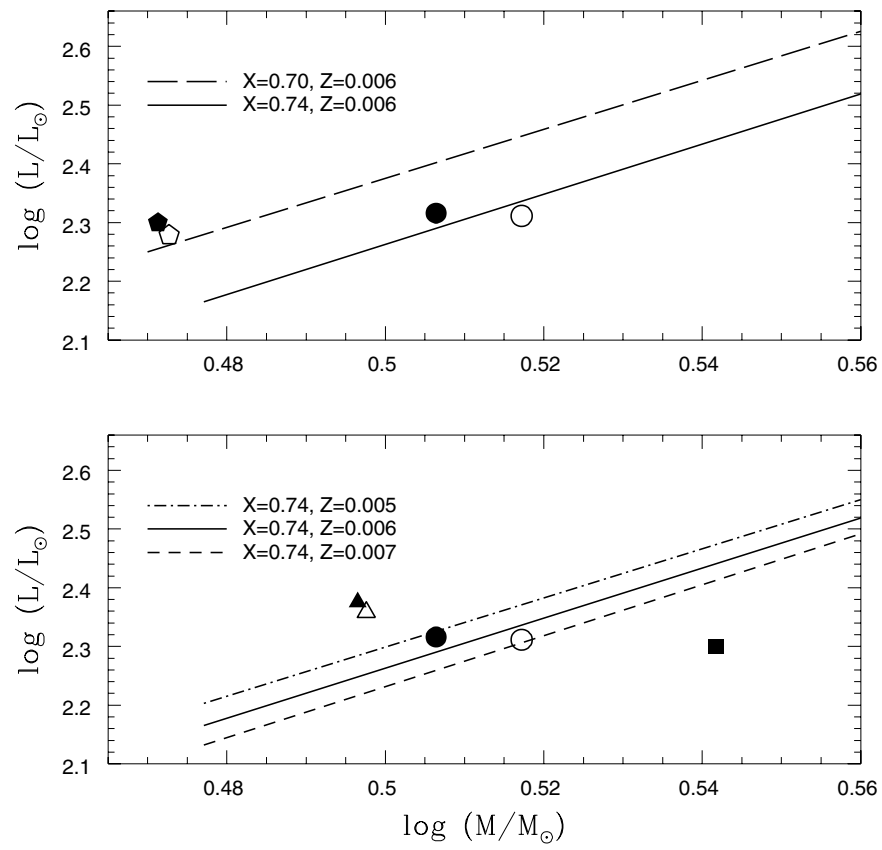

Fig. 2. Evolutionary $M-L$ relation for models with $P_{1}=0.541$. Lines were derived for stellar models undergoing first crossing of the instability strip. Models were calculated assuming no overshooting from the convective core $\left(\alpha_{\mathrm{ov}}=0\right)$ during the main sequence evolution. Symbols represent seismic solutions for Star 1, derived with (nonevolutionary) envelope models (see Table 2). Filled and open symbols correspond to $\alpha_{\text {con }}=1$ and 0 , respectively. Pentagons correspond to $X=0.70, Z=0.006$; circles to $X=0.74, Z=0.006$; triangles to $X=0.74, Z=0.005$; and squares to $X=0.74, Z=0.007$ (there is no solution for $\alpha_{\text {con }}=0$ in the last case).

extend to the instability strip. At $M=3.5 M_{\odot}$ the loop ends within the strip, but then the minimum value of $P_{1}$ is about 1.15. Again, increasing $Z$ only widens the discrepancy.

Determination of the extent of the blue loop is a subtle problem, as shown by wide divergence between various authors. A robust result is the difference of $\Delta \log L \sim 0.2$ between the first and the second crossing of the instability strip by stars in the 3-4 $M_{\odot}$ mass range. The $\log L$ values we found for the triple-mode Cepheids indicate that these two objects are caught during the first crossing of the strip.

\subsection{Models in the first crossing of the instability strip}

We computed a number of evolutionary tracks covering the Hertzsprung gap crossing phase. From each track, we can determine $L$ at the moment when the first overtone period is equal to $P_{1, o}$. The value of $L$ is virtually independent of $\alpha_{\text {con }}$, but depends strongly on $\alpha_{\mathrm{ov}}$, which is the ratio of the overshooting distance from the convective core to the pressure distance scale calculated at the edge of the core. From our model calculations we find

$\left(\frac{\partial \log L}{\partial \alpha_{\mathrm{ov}}}\right)_{M, X, Z, P_{1}} \approx 0.6$.

At specified values of $M, X, Z$ and $P_{1}$, the minimum value of $L$ corresponds to $\alpha_{\mathrm{ov}}=0$.
In Fig. 2 we display the $M-L$ relations for evolutionary models of Star $1\left(P_{1}=0\right.$ d541), calculated with no overshooting. Each line corresponds to a different chemical composition. In the same plot we also display parameters of several seismic models of Star 1, for the same set of the composition parameters (see Table 2). We plot only models corresponding to solution $B$, because only for this solution can agreement between seismic and evolutionary models be reached. The lower panel of the plot explores the effect of varying stellar metallicity. For $Z=0.005$, the seismic models do not agree with $\alpha_{\mathrm{ov}}=0$ evolutionary tracks. The agreement may be easily reached if overshooting of $\alpha_{\mathrm{ov}} \sim 0.1$ is allowed, which shifts the evolutionary $M-L$ relation up. For $Z=0.006$ the seismic models and the evolutionary tracks are almost in agreement, whereas for $Z=0.007$ the seismic model is placed below the corresponding evolutionary $M-L$ line and no agreement between the two is possible. Figure 2 illustrates the general pattern of model behaviour; with $X, \alpha_{\text {con }}$ and $\alpha_{\text {ov }}$ fixed, the exact match between seismic models and evolutionary tracks can be reach only at one specific metallicity value. The matching values of $Z$ become lower, when overshooting distance $\alpha_{\mathrm{ov}}$ is increased.

The upper panel of Fig. 2 shows that a match between seismic and evolutionary models is possible for any reasonable value of $X$, but it yields different values of stellar mass: $M \sim 3.2 M_{\odot}$ for $X=0.74$ and $M \sim 3.0 M_{\odot}$ for $X=0.70$. Interestingly, metallicities of matching models are almost the same independently of $X$.

In Table 4 we list parameters of the two stars, which result from matching seismic models to the evolutionary tracks calculated for $\alpha_{\mathrm{ov}}=0$. The values obtained with two choices of $\alpha_{\text {con }}$ give some assessment of uncertainty due to poor knowledge of convection. We regard the parameters derived at higher $X$ as more likely, because we do not expect any significant $\mathrm{He}$ enrichment for LMC stars with $M \sim 3 M_{\odot}$. In the last two columns we provide the values of $(V-I)$ colour and of the distance modulus to the stars, obtained in same way as in Tables 2 and 3. Bearing in mind uncertainty in the mean dereddened colour, which according to Udalski (private communication) is about $0 \mathrm{~m}$. we regard the agreement in $(V-I)$ to be quite satisfactory. Agreement may be even better, if smaller LMC reddenings of Subramaniam (2005) are used. We will discuss the inferred values of the distance modulus later in this paper. In the last column of Table 4 we list the calculated rates of period change, which will be discussed later, too.

We stress that, except of $\dot{P} / P$, all the values listed in Table 4 were derived from the envelope models. The requirement of matching the seismic envelope models to the evolutionary tracks was used only to a) reject solution $C$; and b) to find the value of $Z$ at which the exact match is achieved.

\subsection{Constraints on the extent of overshooting}

At fixed $X$ and $\alpha_{\text {con }}$, there is a minimum metallicity value allowed by seismic models. This lower limit for $Z$ corresponds to an upper limit for the overshooting distance, $\alpha_{\text {ov }}$. In Table 5 we give the maximum overshooting distances for both triplemode Cepheids. We obtained a stronger constraint for Star 2, 
Table 4. Triple-mode Cepheid models consistent with no overshooting evolutionary tracks.

\begin{tabular}{ccccccccc}
\hline \hline$X$ & $\alpha_{\text {con }}$ & $Z$ & $M / M_{\odot}$ & $\log \left(L / L_{\odot}\right)$ & $\begin{array}{c}\log T_{\text {eff }} \\
{[\mathrm{K}]}\end{array}$ & $\begin{array}{c}(V-I) \\
{[\mathrm{mag}]}\end{array}$ & $\begin{array}{c}m-M \\
{[\mathrm{mag}]}\end{array}$ & $\begin{array}{c}\dot{P} / P \\
{\left[\mathrm{Myr}^{-1}\right]}\end{array}$ \\
\hline \multicolumn{7}{c}{0} & \multicolumn{7}{c}{ Star 1} \\
\hline \multirow{2}{*}{0.70} & 0 & 0.0061 & 2.99 & 2.273 & 3.811 & 0.51 & 18.36 & 1.51 \\
& 1 & 0.0063 & 3.02 & 2.280 & 3.813 & 0.50 & 18.37 & 1.53 \\
& 0 & 0.0057 & 3.25 & 2.323 & 3.818 & 0.48 & 18.43 & 1.65 \\
& 1 & 0.0062 & 3.26 & 2.308 & 3.814 & 0.50 & 18.43 & 1.61 \\
\hline \multirow{2}{*}{0.70} & 0 & 0.0065 & 3.24 & 2.394 & 3.826 & 0.44 & 18.49 & 2.15 \\
& 1 & 0.0069 & 3.26 & 2.392 & 3.826 & 0.44 & 18.49 & 2.18 \\
0.72 & 0 & 0.0061 & 3.39 & 2.432 & 3.832 & 0.42 & 18.53 & 2.11 \\
& 1 & - & - & - & - & - & - & - \\
\hline
\end{tabular}

Table 5. Triple-mode Cepheid models for maximum overshooting allowed by seismic constraints.

\begin{tabular}{cccccccccc}
\hline \hline$X$ & $\alpha_{\text {con }}$ & $Z$ & $\alpha_{\text {ov }}$ & $M / M_{\odot}$ & $\log \left(L / L_{\odot}\right)$ & $\begin{array}{c}\log T_{\text {eff }} \\
{[\mathrm{K}]}\end{array}$ & $\begin{array}{c}(V-I) \\
{[\mathrm{mag}]}\end{array}$ & $\begin{array}{c}m-M \\
{[\mathrm{mag}]}\end{array}$ & $\begin{array}{c}\dot{P} / P \\
{\left[\mathrm{Myr}^{-1}\right]}\end{array}$ \\
\hline \multirow{2}{*}{0.70} & 0 & 0.0043 & 0.33 & 2.81 & 2.393 & 3.845 & 0.36 & 18.34 & 1.69 \\
& 1 & 0.0036 & 0.33 & 2.84 & 2.428 & 3.853 & 0.33 & 18.34 & 1.81 \\
0.74 & 0 & 0.0045 & 0.22 & 3.07 & 2.392 & 3.838 & 0.40 & 18.40 & 1.61 \\
& 1 & 0.0038 & 0.19 & 3.14 & 2.443 & 3.849 & 0.35 & 18.42 & 1.75 \\
\hline \multirow{2}{*}{0.70} & 0 & 0.0056 & 0.12 & 3.18 & 2.450 & 3.841 & 0.38 & 18.49 & 2.07 \\
& 1 & 0.0049 & 0.07 & 3.26 & 2.493 & 3.850 & 0.34 & 18.51 & 2.26 \\
0.72 & 0 & 0.0058 & 0.05 & 3.35 & 2.452 & 3.838 & 0.39 & 18.52 & 2.12 \\
& 1 & - & - & - & - & - & - & - & - \\
\hline
\end{tabular}

especially when the higher value of $X$ was adopted. Despite very similar masses, we cannot argue that the two stars should have similar $\alpha_{\mathrm{ov}}$, because they may well differ in the rotation rate; and rotation induced mixing can mimic the convective overshooting. In any case, the limit of $\alpha_{\mathrm{ov}} \leq 0.12$ obtained for Star 2 is very interesting.

The triple-mode Cepheids can be quite important for constraining element mixing in moderate mass stars. To demonstrate this point, Table 5 also lists the stellar parameters of the two Cepheids derived at the extreme values of $\alpha_{\mathrm{ov}}$. These can be compared with parameters derived for $\alpha_{\mathrm{ov}}=0$ (Table 4). As overshooting is increased, the matching models become less metal abundant and bluer. Thus, the limits on the extent of overshooting can be further improved once we precisely measure either metallicities or the mean dereddened colours of the two stars. Here, we make a natural assumption that the photospheric composition is representative for the whole period forming envelope.

\section{The distance to LMC}

The values of distance moduli listed in Tables 4 and 5 are all between 18.34 and 18.53 , i.e. well within the range of values currently considered for the LMC. They are indeed very close to estimates based on analysis of RRd stars, which yields $18 \mathrm{~m} 53$ according to Kovács (2000b) and $18 \mathrm{~m} .42$ according to Popielski et al. (2000). From the double-mode Cepheid data, Kovács (2000a) obtained the value of 18 m 54 . All these determinations rely on fitting the observed pulsation periods, although for different objects and in different ways.

For the same model parameters $\left(X, \alpha_{\text {con }}\right)$, there is a systematic difference of about 0.13 between distance moduli derived for Star 1 and Star 2. We believe that a large part of this difference can be attributed to $W_{I}$ measurement errors. Judging from faint Cepheids $\left(W_{I}>15\right.$. 0 ) with independent photometry from two OGLE fields, the error of the mean Wessenheit index is indeed about 0.06 on average, but sometimes can exceed 0.09 . We cannot exclude, however, that some part of the 0.13 difference might be real. As shown by Subramaniam (2003), the $m-M$ displays considerable variation even within the LMC bar.

We want to stress that distances of the triple-mode Cepheids can be estimated very accurately once photometric precision is improved. As seen from Tables 4 and 5, the total range of $m-M$ allowed by seismic models is only 0 . 09 for Star 1 and 0 . 04 for Star 2. On principle, the individual distances of the two stars can be determined with, respectively, $\pm 2 \%$ and $\pm 1 \%$ accuracy. 


\section{Period changes}

The first crossing of the instability strip is faster by two orders of magnitude than either the second or the third crossing. Measuring the rates of period change should, therefore, provide a crucial test for the triple-mode Cepheid models.

The rates of period change, $\dot{P} / P$, inferred from our models are given in the last column of Tables 4 and 5. They are about $2 \mathrm{Myr}^{-1}$, somewhat below this value for Star 1 and somewhat above it for Star 2. The rates are calculated for the first overtone, but are approximately the same for all modes. With such values of $\dot{P} / P$, the $(\mathrm{O}-\mathrm{C})$ should reach $6-10 \%$ of the pulsation period in ten years.

We do not yet have ten years worth of data at our disposal, but we will try to measure $\dot{P}$ with the photometry already at hand. Our method consists in the least square fitting of photometric measurements with the Fourier sum of the form

$$
\begin{array}{r}
I(t)=\langle I\rangle+\sum_{i=1}^{3} \mathrm{~A}_{i} \cos \left[\left(\omega_{i}+\frac{1}{2} \dot{\omega}_{i} t\right) t+\phi_{i}\right] \\
+ \text { harmonics }+ \text { combination terms }
\end{array}
$$

where $\omega_{i}=2 \pi / P_{i}$ are pulsation frequencies and $\dot{\omega}_{i}=$ $-2 \pi \dot{P}_{i} / P_{i}^{2}$ are the rates of frequency change (see e.g. Kepler 1993, for derivation). The nonlinear least square fit is performed with the Marquardt algorithm (Press et al. 1986). In the following, we will discuss only the rates of period change for the first overtone. The other two modes have much lower amplitudes and consequently their $\dot{P}$ is determined with 3-10 times greater error.

The analysis of DIA-reduced OGLE $I$-band photometry yields $\dot{P}_{1} / P_{1}=+1.7 \pm 3.7 \mathrm{Myr}^{-1}$ for Star 1 and $\dot{P}_{1} / P_{1}=$ $+5.3 \pm 6.4 \mathrm{Myr}^{-1}$ for Star 2. Clearly, the OGLE-II timebase of $\sim 1200$ day is not long enough to obtain significant $\dot{P}$ measurement.

A considerably longer timebase ( 2700 day) is offered by MACHO photometry (Allsman \& Axelrod 2001). Both triplemode Cepheids have been observed by the MACHO Project (LMC SC3-360128= MACHO 77.8029.310; LMC SC5$338399=$ MACHO 80.7074.120) and over 1500 measurements have been accumulated for each of them. Unfortunately, MACHO data are of much lower quality. We decided to perform initial filtering of MACHO photometry by rejecting a) all outlying measurements deviating by more than $5 \sigma$ from average brightness of the star and b) all measurements with photometric errors (given by MACHO) larger than 5 times the average for the dataset. Further data clipping was done during the fitting procedure, when all points deviating by more than $5 \sigma$ from the fit were also rejected. For the final determination of $\dot{P}$ we used the MACHO $B$-band photometry. The analysis yields

$\dot{P}_{1} / P_{1}=-1.66 \pm 0.83 \mathrm{Myr}^{-1}$ for Star 1

$\dot{P}_{1} / P_{1}=-2.28 \pm 0.94 \mathrm{Myr}^{-1}$ for Star 2 .

For both Cepheids, $\dot{P} / P$ is determined only with $2 \sigma$ accuracy. Therefore, we do not consider the above values to be significant detections, but rather to provide observational limits on $\dot{P}$. The results, nevertheless, are very interesting, because these limits seem to conflict with the model predictions. The measured $\dot{P} / P$ values differ from theoretical ones (Tables 4 and 5) by $3.8 \sigma$ for Star 1 and by $4.6 \sigma$ for Star 2. If real, such a discrepancy would mean serious trouble for the evolutionary models; however, we should take this result with a grain of salt. The quoted errors of $\dot{P} / P$ are formal statistical errors resulting from the nonlinear least square procedure, which often underestimates the true uncertainties of the fitted parameters. This is especially true in the case of multiperiodic pulsations (Costa et al. 1999; Costa \& Kepler 2000).

The $\dot{P}$ determinations reported in this section require confirmation with better data, which will soon be possible when OGLE-III photometry is released. Combined OGLE-II+III dataset will provide a timebase similar to that of MACHO, but much higher data quality should result in $\dot{P}$ errors about twice as low.

More data should eventually lead to determining $\dot{P}$ for all three modes, which is essential for understanding the true nature of the period changes. If they are caused by stellar evolution, then all modes should yield roughly the same values of $\dot{P} / P$, while no such equality is expected if period changes result from nonlinear mode interactions.

\section{Conclusions and discussion}

We show that pulsation periods measured in two LMC triplemode Cepheids are consistent with values calculated for the first three overtones only if the envelope metal abundance is between $Z=0.004$ and 0.007 . This is somewhat lower than the $Z=0.008$ typically adopted for the LMC. In one of the stars, fitting the periods requires the envelope hydrogen abundance $X \lesssim 0.72$, which is again somewhat below the commonly accepted LMC value of $X=0.74$. Conclusions regarding envelope chemical composition rest mainly on the measured period ratios. For calculating the model periods, we rely on the linear pulsation theory and believe that nonlinear corrections to the periods are small, but we cannot estimate them. Therefore, it is important that the derived metallicity limits are verified through direct spectroscopic determination.

Effective temperatures implied by the models are consistent with observed $(V-I)$ colours of the two stars. Unfortunately, the accuracy of the latter is currently too low to yield useful constraints. Our models have luminosities confined to narrow ranges. The inferred distance moduli are $18 \mathrm{~m} .34-18 \mathrm{~m} .43$ for one star and 18.49-18. 53 for the other. The difference between the two objects can be in large part attributed to photometric errors. The derived distances to the triple-mode Cepheids are within the range currently considered for the LMC, but favour longer LMC distances in this range.

We find that parameters inferred from the pulsation periods of the two stars are consistent with evolutionary models undergoing the Hertzsprung gap crossing. This consistency does not require any overshooting from the convective core during the main sequence evolution. Seismic models do not exclude overshooting, though, but instead impose an upper limit on its extent. The limit is particularly strong in the case of LMC SC5-338399. The extent of overshooting can be 
further constrained once metal abundances or, alternatively, mean dereddened colours of the stars are precisely measured.

Both the periods and the luminosities of the triple-mode Cepheids cannot be reconciled with evolutionary models in the phase of central helium burning. Thus, these stars must be in the phase of Hertzsprung gap crossing. Such an interpretation does not imply an unlikely coincidence. Stars with masses of $\sim 3 M_{\odot}$, which we find for our objects, spend about $10^{5}$ years crossing the instability strip on their way to the red giant branch, which is comparable to time spent inside the strip by more massive stars $\left(M \sim 7 M_{\odot}\right)$ during the central helium burning phase. The pulsation periods of such stars are in the 10 to 20 days range, which is well populated by LMC Cepheids.

What may be a problem for the first crossing scenario is a possible conflict between predicted and measured rates of period change. The currently available data are insufficient for significant $\dot{P}$ determination. We note, however, that in both Cepheids the most probable $\dot{P}$ values are more than $3.8 \sigma$ away from theoretical expectations. Since we are somewhat sceptical of $\dot{P}$ measurement errors, we do not yet consider this result to invalidate our models, but it is a warning sign.

If future determinations confirm the discrepancy, our models will be in serious trouble. In our opinion, this problem would be more worrisome than the one encountered by Pietrukowicz (2003) for long period Cepheids. He found that theoretically predicted rates of period change are faster than the measured ones, but the size of the effect depends on whose evolutionary tracks are used. According to our unpublished calculations, there is no significant conflict if one uses the tracks of Girardi et al. (2000).

The speed of evolution during the second and third crossings of the instability strip is affected by element mixing in three distinct evolutionary phases. There are uncertainties regarding mixing efficiency in each of them. During the main sequence phase, we do not know the extent of convective overshooting from the hydrogen burning core. During the red giant phase, we do not know the efficiency of the dredge up of the helium enriched material into the deep convective envelope. Finally, during the blue loop phase, there is a subtle problem with treating the boundary of the helium burning core. In the case of the first crossing of the instability strip, only the uncertainty of the main sequence overshooting affects the results, and this was taken into account in our calculations. We believe that our prediction for the rates of period change is trustworthy; therefore, the possible discrepancy between calculated and observed $\dot{P}$ would pose a very serious challenge to our understanding of stellar evolution. Further observations of both triple-mode Cepheids are needed to settle this important issue.

Acknowledgements. This work has been supported in part by Polish KBN grant 5 P03D 030 20. The authors wish to thank A\&A Language Editor, J. Adams, for many helpful suggestions which have improved this paper.

\section{References}

Alibert, Y., Baraffe, I., Hauschildt, P., \& Allard, F. 1999, A\&A, 344, 551

Allsman, R. A., \& Axelrod, T. S. 2001 [arXiv: astro-ph/0108444]

Costa, J. E. S., \& Kepler, S. O. 2000, Baltic Astron., 9, 451

Costa, J. E. S., Kepler, S. O., \& Winget, D. E. 1999, ApJ, 522, 973

Dziembowski, W. 1977, Acta Astron., 27, 95

Fitch, W. S., \& Szeidl, B. 1976, ApJ, 203, 616

Fokin, A. B. 1994, A\&A, 292, 133

Girardi, L., Bressan, A., Bertelli, G., \& Chiosi, C. 2000, A\&A, 354, 892

Iglesias, C. A., \& Rogers, F. J. 1996, ApJ, 464, 943

Kepler, S. O. 1993, Baltic Astron., 2, 515

Kovács, G. 2000a, A\&A, 360, L1

Kovács, G. 2000b, A\&A, 363, L1

Kovács, G., \& Buchler, J. R. 1988, ApJ, 324, 1026

Kovács, G., \& Buchler, J. R. 1994, A\&A, 281, 749

Kurucz, R. L. 1998, http://kurucz . harvard.edu

Madore, B. F., \& Freedman, W. L. 1991, PASP, 103, 933

Moskalik, P., \& Buchler, J. R. 1991, ApJ, 366, 300

Moskalik, P., \& Kołaczkowski, Z. 2005, in preparation

Moskalik, P., Kołaczkowski, Z., \& Mizerski, T. 2004, in Variable Stars in the Local Group, ed. D. W. Kurtz, \& K. Pollard, ASP Conf. Ser., 310,498

Paczyński, B. 1970, Acta Astron., 20, 47

Pietrukowicz, P. 2003, Acta Astron., 53, 63

Popielski, B. L., Dziembowski, W. A., Cassisi, S. 2000, Acta Astron., 50, 491

Press, W. H., Teukolsky, S. A., Vetterling, W. T., \& Flannery, B. P. 1986, Numerical Recepies in FORTRAN (2nd ed.; Cambridge: Cambridge Univ. Press)

Rogers, F. J., Swenson, F. J., \& Iglesias, C. A. 1996, ApJ, 456, 902

Soszyński, I., Udalski, A., Szymański, M., et al. 2000, Acta Astron., 50,451

Subramaniam, A. 2003, ApJ, 598, L19

Subramaniam, A. 2005, A\&A, 430, 421

Udalski, A., Soszyński, I., Szymański, M., et al. 1999, Acta Astron., 49, 223

Żebruń, K., Soszyński, I., Woźniak, P. R., et al. 2001, Acta Astron., 51,317 